\title{
The association between differentially expressed micro RNAs in breast cancer cell lines and the micro RNA-205 gene polymorphism in breast cancer tissue
}

\author{
JINGCHENG ZHANG ${ }^{1}$, BIN WEI ${ }^{1}$, HUIXIAN HU ${ }^{1}$, FANRONG LIU ${ }^{2}$, YAN TU ${ }^{1}$ and FANG HE ${ }^{1}$ \\ ${ }^{1}$ Department of Hematology, Jinhua Hospital of Zhejiang University, Jinhua, Zhejiang 321000; ${ }^{2}$ Department of Pathology, \\ Second Hospital Affiliated to Nanchang University, Nanchang, Jiangxi 330006, P.R. China
}

Received December 14, 2015; Accepted October 26, 2017

DOI: $10.3892 / \mathrm{ol} .2017 .7550$

\begin{abstract}
Micro (mi)RNAs are an endogenous non-coding small RNA comprised of 19-26 nucleotides. miRNAs regulate gene expression through the recognition of its 'seed sequence' and interactions with 3'-untranslated region of target miRNAs. Previous studies identified that miRNAs are associated with the onset and development of breast cancer and that a number of mutations in the coding DNA sequence of miRNAs affect its expression. Therefore, the present study aimed to screen differentially expressed miRNAs using miRNA expression profile chips to analyze the expression of miRNA (miR)-205 in 12 breast cell lines of different metastatic performance and benign proliferative variation as well as breast cancer via in-situ hybridization, and screen out single-nucleotide polymorphisms (SNPs) in the miR-205 coding gene region. In addition, the association between SNP and the clinicopathological features of breast lesions was investigated. The results of the present study demonstrated that the expression of miR-205 was decreased in breast cancer cells and tissues compared with benign lesions. SNPs in the miR-205 coding gene region were identified in tumor cell lines and as increasing lengths of the AGC repetitive sequence were lost, miR-205 expression increased. An association was identified between a number of SNPs in the miR-205 coding gene region and breast cancer, as well as between SNPs in miR-205 coding gene region and the clinicopathological features of breast cancer.
\end{abstract}

\section{Introduction}

Single nucleotide polymorphisms (SNPs) are important in determining the pathogenesis of various diseases, identifying

Correspondence to: Ms. Fang He, Department of Hematology, Jinhua Hospital of Zhejiang University, 365 People East Road Road, Jinhua, Zhejiang 321000, P.R. China

E-mail: 16995722@qq.com

Key words: micro RNA-205, breast cancer, single nucleotide polymorphism, clinical pathology effective therapeutic targets and screening individualized chemotherapy programs. A number of in-depth studies have explored SNP variations in coding genes and the influence of this on the incidence and development of human cancer $(1,2)$. However, research investigating SNPs in non-coding micro (mi)RNA genes and their effects on breast cancer progression remain in the early stages (3).

MiRNAs are involved in a number of important biological processes, from embryonic development to the evolution of various diseases. These processes are often accompanied by the abnormal expression of various miRNAs and their target genes, with previous studies having demonstrated that miRNA expression is dysregulated in cancer, and experimental data having indicated that cancer phenotypes may be modified by targeting miRNA expression (4). It has been demonstrated that SNPs arising in miRNA coding gene sequences affect the synthesis of miRNA, whereas SNPs in miRNA target gene sequences affect the interaction between miRNA and its target genes, thus affecting the regulatory function of miRNAs (5). The polymorphism of miRNA (miR)-323b (4) is associated with hepatitis $\mathrm{B}$ virus replication, whereas the variation of rs4846049 in the 3'-untranslated region (UTR) of 5,10-methylenetetrahydrofolate reductase affects the binding of miRNA to increase the risk of coronary heart disease (6). Similar studies have been conducted in other types of cancer, including gastric and lung cancer $(7,8)$.

SNP variation in miRNA coding genes is associated with the onset and progression of breast cancer. It has been demonstrated that variation at the miR-27a rs895819 site may affect miR-27a expression: Compared with carriers of A-alleles, G-allele carriers have a significantly reduced risk of developing breast cancer $(\mathrm{P}=0.032)(9)$. Binding sites from miR-515-5p exist in the 3'-UTR of the insulin-like growth factor 1 receptor gene; variation in the rs 28674628 site in this region affects the binding of miR-515-5p to the gene, thus increase the risk of breast cancer (10). SNP variations of miRNAs, including miR-192-a 2 and miR-423 are also associated with breast cancer risk $(11,12)$.

The present study aimed to provide a theoretical basis for the exploration of SNPs in miRNA coding genes to be applied in clinical practice as molecular markers. This would entail the SNP screening of miRNA coding genes associated with breast 
cancer metastasis. The association between SNP sites in miRNA coding genes and the incidence and development of breast cancer in clinical specimens was also investigated in the current study.

\section{Materials and methods}

Cells. The highly malignant human breast cancer cell lines BT549, HS578T, MDA-MB-231 and SUM159PT, and the less malignant human breast cancer cell lines BT474, MCF7, MDA-MB-468, SK-BR-3, T47D and ZR-75-1, as well as the immortalized normal human breast cell lines MCF10A and MCF12A were acquired as gifts from Professor Shi Huidong, Cancer Research Center of Medical College of Georgia (Augusta, GA, USA). The human lung cancer cell lines 95-D and A549, human stomach cancer cell lines AGS and MGC-803, and the human hepatocarcinoma cell lines SMMC-7721 were purchased from the Cell Bank of the Chinese Academy of Sciences (Shanghai, China). Tissue chips of human breast diseases were acquired from Alenabio (Xi'an, China; cat. no. BR723). The chips included 83 cases of breast cancer and 35 cases of breast lobular hyperplasia in a dual-chip matrix. The tissue samples were surgically resected during surgery and fixed for $24 \mathrm{~h}$ with $10 \%$ neutral-buffered formaldehyde, with a dot diameter of $1.1 \mathrm{~mm}$ and a thickness of $4 \mu \mathrm{m}$. A total of 60 human breast surgical resection samples were obtained from the Department of Pathology of Jinhua Hospital of Zhejiang University (Jinhua, China) between March 2010 to August 2012. All patients were female with a mean age of 62 years (range, 32-79 years). Written informed consent was obtained from all patients for the use of their samples.

Analysis of expression profile chip of miRNA. Total RNA from cell lines was extracted using the RNeasy kit (Qiagen, Inc., Valencia, CA, USA) and RNase-Free DNase Set (Qiagen, Inc.), according to the manufacturer's protocols. Expression profiles of extracted RNA from all cell lines were analyzed using a GeneChip miRNA 2.0 array (Affymetrix; Thermo Fisher Scientific, Inc.). A FlashTag Biotin RNA Labeling kit (Genisphere, Hatfield, PA, USA) was used to perform biotin labeling of $1 \mu \mathrm{g}$ miRNA. Chip hybridization, elution, image acquisition and data standardization were conducted according to the manufacturer's protocol (Fluidics Protocol FS450_0003; Affymetrix; Thermo Fisher Scientific, Inc.). Partek Genomic Suite v.6.2 software (Partek Inc., St. Loius, MI, USA) was used to analyze the chip data.

Reverse transcription-quantitative polymerase chain reaction $(R T-q P C R)$. First-strand complementary DNA (cDNA) was synthesized with oligo-dT or random hexamers as primers, using the SuperScript First-Strand Synthesis System (Invitrogen; Thermo Fisher Scientific, Inc.) according to the manufacturer's protocol. An equal volume mixture of cDNA products (50 ng) were used as templates for PCR amplification. QPCR reactions were performed in a $25 \mu l$ volume with $\mathrm{iQ}^{\mathrm{TM}}$ SYBR Green Supermix (Bio-Rad Laboratories, Inc., Hercules, CA, USA), and $200 \mathrm{nM}$ each of forward and reverse primers using an iCyler iQ instrument and iQ software (Version 2.0; Bio-Rad Laboratories, Inc.). Each sample was analyzed in triplicate. QPCR cycling conditions included an initial denaturation step of $1 \mathrm{~min}$ at $95^{\circ} \mathrm{C}$, followed by 40 cycles consisting of $30 \mathrm{sec}$ at $95^{\circ} \mathrm{C}, 5 \mathrm{sec}$ at $95^{\circ} \mathrm{C}$ and $30 \mathrm{sec}$ at $60^{\circ} \mathrm{C}$. Mean quantification cycle $(\mathrm{Cq})$ values from the triplicate $\mathrm{qPCRs}$ for a gene of interest (GOI) were normalized against the average $\mathrm{Cq}$ values for GAPDH from the same cDNA sample (13). The following primers were used: miR-205 forward, 5'-ACAGGC TGAGGTTGACATGC-3'; and reverse, 5'-GAGTTACTCTTG CTGCTGCTG-3'; GAPDH forward, 5'-TGCACCACCAAC TGCTTAGC-3'; and reverse, 5'-GGCATGGACTGTGGTCAT GAG-3'.

Situ hybridization. Highly malignant human breast cancer cell lines BT549, MDA-MB-231 and low malignant human breast cancer cell lines MCF7 and T47D were used in the subsequent experiment. Cells were grown on coverslips for 2-3 days, and the coverslips were washed with PBS at $37^{\circ} \mathrm{C}, 3$ times. Cells were fixed with $4 \%$ neutral formalin for $5 \mathrm{~min}$, and incubated with operating fluid of proteinase $\mathrm{K}(0.25 \mathrm{mg} / \mathrm{ml}$ in DEPC H2O-HCl; Sigma Aldrich; Merck KGaA), at $37^{\circ} \mathrm{C}$ for dissociation after $15 \mathrm{~min}$, followed by situ hybridization operation. Breast cancer and breast benign lesions (fibroadenoma, breast hyperplasia), sectioned at $5-\mu \mathrm{m}$ onto tissue slides were deparaffinized, treated with $0.2 \mathrm{~N} \mathrm{HCl}$, incubated in $1 \mathrm{M}$ sodium thiocyanate and immersed in protease solution (Vysis Inc., Downers Grove, IL, USA) for 10 min at $37^{\circ} \mathrm{C}$. The tissues were then fixed with $10 \%$ formalin for $10 \mathrm{~min}$, denatured for $5 \mathrm{~min}$ at $72^{\circ} \mathrm{C}$ and sequentially incubated in 75, 90 and $100 \%$ ethanol. The tissues were then treated with proteinase $\mathrm{K}$ for $6 \mathrm{~min}$ at $37^{\circ} \mathrm{C}$, followed by their dehydration and hybridization. The hybridization mixture contained the labeled oligonucleotide probe, $50 \%$ formamide, $10 \mathrm{mmol} / \mathrm{l} \mathrm{Tris-HCl,} 1 \mathrm{mmol} / \mathrm{l}$ vanadyl-ribonucleoside complex (94740; Sigma Aldrich; Merck KGaA), 1 mmol/l CTAB (855820; pH 7.0; Sigma Aldrich; Merck KGaA), 0.15 mol/1 $\mathrm{NaCl}, 1 \mathrm{mmol} / \mathrm{E}$ EDTA (pH 7.0), $1 \mathrm{X}$ Denhardt's mixture and $10 \%$ dextran sulfate. Subsequent to hybridization, the slides were washed 3 times, for $30 \mathrm{~min}$ each time, in $0.1 \mathrm{~mol} / \mathrm{l}$ Tris-buffered saline (TBS) at room temperature. The slides were then treated with TBS $[100 \mathrm{mmol} / \mathrm{l}$ Tris $(\mathrm{pH} \mathrm{7.5)}$ and $150 \mathrm{mmol} / 1 \mathrm{NaCl}$ ] containing a $1 \%$ blocking reagent (Roche Diagnostics, Shanghai, China) and $0.03 \%$ Triton X-100 for $30 \mathrm{~min}$ at room temperature, and incubated for $30 \mathrm{~min}$ with anti-dioxigenin alkaline phosphatase-conjugated antibodies (Roche Diagnostics) diluted at 1:4,000 in TBS containing 0.03\% Triton X-100 and a $1 \%$ blocking reagent. Subsequent to being washed three times, for $15 \mathrm{~min}$ in TBS and $0.05 \%$ Tween 20, the slides were rinsed in a diammonimum phosphate (DAP)-buffer [100 mmol/l Tris (pH 9.5) $100 \mathrm{mmol} / \mathrm{l} \mathrm{NaCl}, 50 \mathrm{mmol} / \mathrm{l} \mathrm{MgCl} 2]$ and hybridization signals were subsequently visualized using nitroblue tetrazolium and 5-bromo-4-chloro-3-indolyl phosphate as substrates [DAP-buffer $(100 \mathrm{mmol} / \mathrm{l}$ Tris, $\mathrm{pH} 9.5$, $100 \mathrm{mmol} / \mathrm{l} \mathrm{NaCl}$ and $50 \mathrm{mmol} / \mathrm{l} \mathrm{MgCl} 2$ ) in 10\% PVA (341584; Sigma Aldrich)].

Extraction of DNA from paraffin-embedded tissues. DNA was extracted from paraffin-embedded breast cancer and breast benign lesions (fibroadenoma, breast hyperplasia) tissues. Areas of the tissue and cytology slides containing the tumor cells of interest were marked by a cytopathologist using a pen. A diamond-tipped pencil was then used to mark the underside of the slide. Following removal of the cover slip, tumor cells were scraped with a 26-gauge needle. Briefly, 50-100 $\mu 1$ of 
DNA extraction buffer solution (50 mM Tris buffer, $\mathrm{pH} 8.3$; $1 \mathrm{mM}$ EDTA, pH 8.0; 5\% Tween-20 and $200 \mu \mathrm{g} / \mathrm{ml}$ proteinase $\mathrm{K})$ with $10 \%$ resin was added to the scraped cells. Following incubation at $56^{\circ} \mathrm{C}$ for $\sim 1 \mathrm{~h}$, the tube was heated to $100^{\circ} \mathrm{C}$ for $20 \mathrm{~min}$ followed by centrifugation at $900 \mathrm{xg}$ for $10 \mathrm{~min}$ at $4^{\circ} \mathrm{C}$ to pellet the debris. The recovered supernatant was used for the PCR.

DNA sequencing. Amplified specimens processed by PCR were sent to Sangon Biotech Co., Ltd., (Shanghai, China) for sequencing and the genotypes of specimens were analyzed using the obtained sequence diagram. The obtained sequence was analyzed using the 2014 UCSC Genome Browser (http://genome.ucsc.edu) (14).

Statistical analysis. Data was analyzed using SPSS 20.0 (IMB Corp., Armonk, NY, USA). Student's t-test was performed for between-group comparisons, $\chi^{2}$ for comparisons between count data rate and correlations were determined using Pearson's correlation analysis. $\mathrm{P}<0.05$ was considered to indicate a statistically significant difference.

\section{Results}

Analysis of miRNA expression profile chip in breast cancer cells with more or less metastasis. A total of 4 highly metastatic breast cancer cell lines and 6 less metastatic breast cancer cell lines, as well as 2 immortalized cell lines were selected to determine miRNA expression profiles. Following data processing and statistical analysis of chip results, it was identified that there were 9 differentially expressed miRNAs among the 12 cell lines $(\mathrm{P}<0.01$, numerical value $\geq 20$ or $\leq-20$ ). Compared with the less metastatic group, levels of miR-100, miR-138 and miR-146a were increased in the highly metastatic cell lines, whereas levels of miR-375, miR-34a, miR-141, miR-203, miR-205 and miR-200c were decreased in the highly metastatic group (Fig. 1A).

The expression of miR-205 in more and less metastatic breast cancer cells. A total of 8 breast cancer cell lines with different metastatic properties underwent RT-qPCR to measure the expression of miR-205. The results were consistent with the aforementioned miRNA chip data, whereby miR-205 was expressed in increased amounts in the less metastatic cell lines compared with more metastatic cell lines $(\mathrm{P}<0.05$; Fig. $1 \mathrm{~B}$ and $\mathrm{C})$.

miR-205 expression in breast cancer cells and tissue chips measured by in-situ hybridization. In-situ hybridization was performed to analyze the expression of miR-205 following the fixation of 4 breast cancer cell lines with formalin; the concentration of miR-205 of the digoxigenin-labelled cDNA probe was $100 \mathrm{nmol} / \mathrm{l}$. The results revealed that the expression of miR-205 in BT549 and MDA-MB-231 was decreased compared with that in MCF7 and T47D (Fig. 2A). The presence of blue or blue purple granules located in breast glandular epithelium or cancer cell cytoplasm and karyon indicated the positive expression of miR-205, and blue or blue purple granules located in karyon of breast ductal epithelium or mesenchymal cells indicated the positive expression of U6.
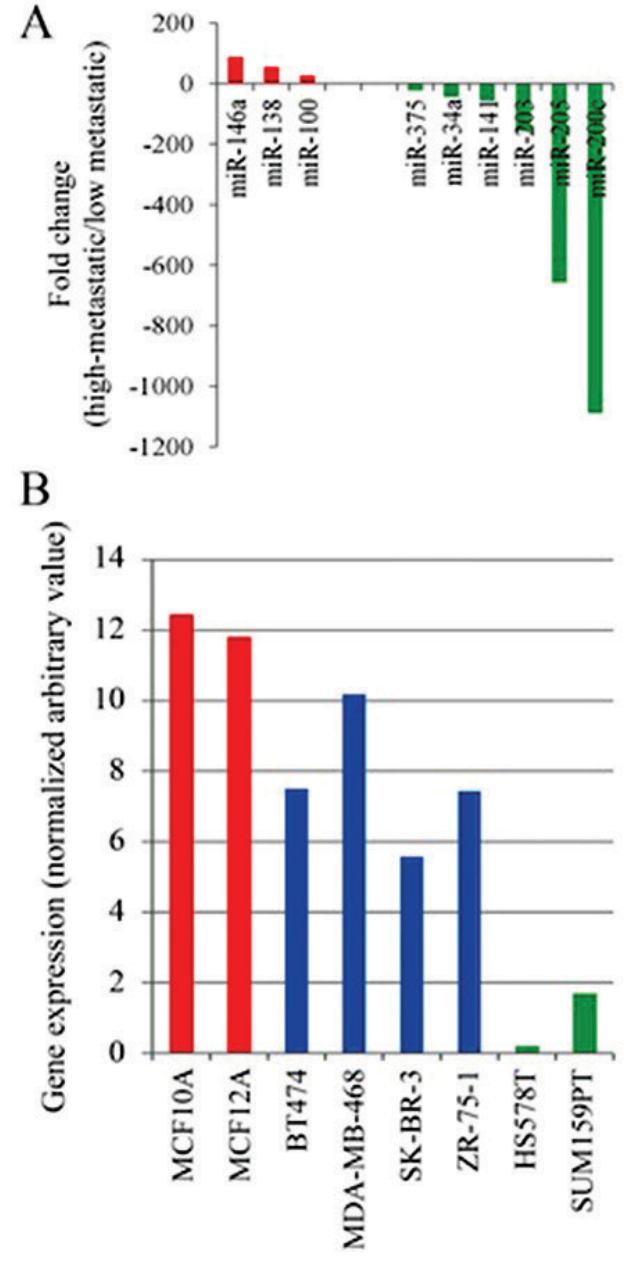

C Normal $\begin{gathered}\text { Less- } \\ \text { aggressive }\end{gathered}$ Aggressive

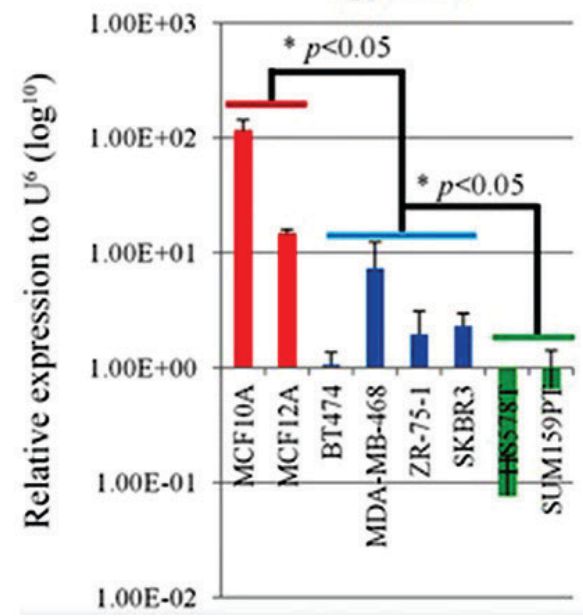

Figure 1. Analysis of miRNA expression in more and less metastatic breast cancer cells. (A) Analysis of miRNA expression profile chip in more and less metastatic breast cancer cell lines. There were 9 miRNA with differential expression in within the 12 cell lines $(\mathrm{P}<0.01$, numerical value $\geq 20$ or $\leq-20)$. Compared with the less metastatic group, the expression of miR-100, miR-138 and miR-146a were increased whereas the expression of miR-34a, miR-141, miR-200c, miR-203, miR-205 and miR-375 were decreased in the more metastatic group. (B) Analysis of the miR-205 expression profile chip in the more and less metastatic breast cancer cell lines. (C) Expression of miR-205 in more and less metastatic breast cancer cells was detected by reverse transcription-qualitative polymerase chain reaction. miR-205 expression was decreased in more metastatic cancer cell lines compared with less metastatic cell lines $(\mathrm{P}<0.05)$ and miR-205 expression in less metastatic breast cancer cell lines was decreased compared with that in normal cells $(\mathrm{P}<0.05)$. miR, microRNA. 
A

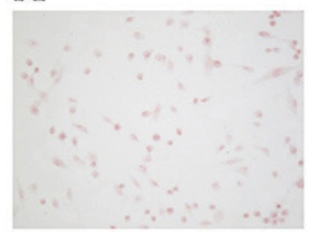

BT549

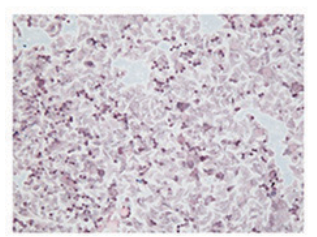

MCF7

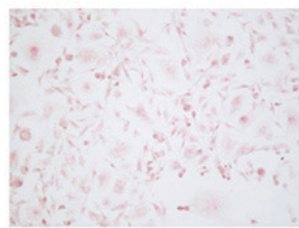

MDA-MB-231

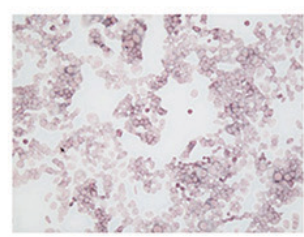

T47D
B
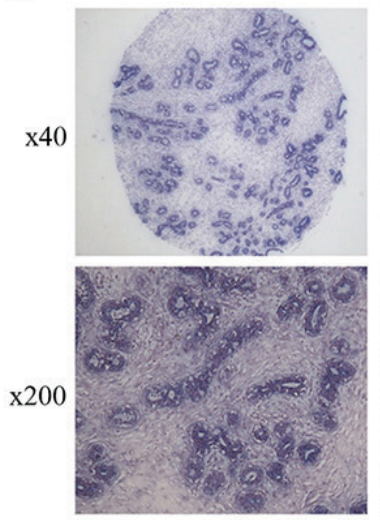

B8 fibroadenoma
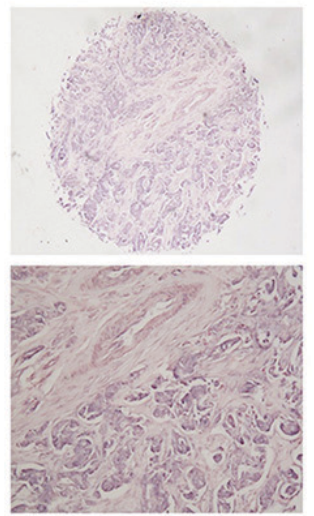

E9 ductal carcinoma

Figure 2. Expression of miR-205 in breast cancer cells and tissue chips was detected using in-situ hybridization. (A) Detection of the expression of miR-205 in breast cancer climbing film cells. The expression of miR-205 in the more metastatic BT549 and MDA-MB-231 cell lines was decreased compared with the less metastatic MCF7 and T47D cell lines. (B) Detection of miR-205 expression in breast tissue chips using in-situ hybridization. MiR-205 probe hybridization identified positive expression (blue purple granules) located in breast ductal epithelium or cancer cell cytoplasm and karyon, miR-205 is highly expressed in breast fibroadenoma (B8) than ductal carcinoma (E9).

Following the in-situ hybridization of breast normal tissues, no blue granules were exhibited; the results of the U6 probe hybridization revealed positive signal of blue purple granules located in karyon of breast ductal epithelium or mesenchymal cells (Fig. 2B). The results of the miR-205 probe hybridization revealed a positive signal with blue purple granules located in the breast ductal epithelium or cancer cell cytoplasm and karyon (Fig. 2B). In the 36 cases of normal and benign breast lesions, 33 cases $(91.67 \%)$, indicating positive expression (+) of miR-205; in the 36 cases of breast cancer, 23 cases $(63.89 \%)$. MiR-205 expression in benign breast lesions was deemed to be significantly higher than in breast cancer tissue (Table I; $\mathrm{P}=0.011$ ). The expression of miR-205 was not associated with tumor-node-metastasis stage or clinical stage of breast cancer.

Analysis of PCR amplification and sequencing of miR-205. A total of 10 cell lines were cultured for this experiment, including 4 breast cancer cell lines (BT-549, MDA-MB-231, MCF7 and T47D), 2 lung cancer cell lines (95-D and A549), 2 stomach cancer cell lines (AGS and MGC-803) and a liver cancer cell line (SMMC-7721). Cells were collected and RNA and DNA were extracted separately. The SinoBio PCR system was used to amplify miR-205 genes and the electrophoresis results of amplified products are presented in Fig. 3A. Fig. 3B demonstrates the site map of SNPs of the miR-205 gene as determined using the UCSC Genome Browser. Amplified products were sent to Sangon Biotech for sequencing. Sequencing results revealed that miR-205-rs3842642 was present in a number of these 10 cell lines and was broadly divided into four types (Fig. 3C-E); 9/9 homozygous genotype, 13/13 homozygous genotype, 9/13 heterozygous genotype and 7/9 special genotype. Among them, AGS, BT-549 and MCF7 belong to the 9/9 homozygous genotype, 95-D, A549, MGC-803, HEPG2 and SMMC-7721 belong to the 9/13 heterozygous genotype, MDA-MB-231 is a 13/13 homozygous genotype and T47D is a special genotype.

Expression of miR-205 in specimens of different rs 3842642 polymorphisms. DNA was extracted from paraffin-embedded
Table I. Analysis of the expression of miR-205 in normal, benign breast and breast carcinoma.

\begin{tabular}{lccccc}
\hline \multicolumn{5}{c}{$\begin{array}{c}\text { Positive } \\
\text { miR-205 } \\
\text { Case }\end{array}$} & \multicolumn{5}{c}{$\begin{array}{l}\text { expression, } \\
\mathrm{n}(\%)\end{array}$} & P-value & $\chi^{2}$ \\
\hline Normal, benign breast & 36 & $33(91.67)$ & $0.011^{\mathrm{a}}$ & 6.509 \\
Breast carcinoma & 23 & $23(63.89)$ & & \\
\hline
\end{tabular}

${ }^{\mathrm{a}} \mathrm{P}<0.05$.

breast cancer specimens using xylene solvent. These fragments were then amplified using PCR and sent to Sangon Biotech for sequencing. By analyzing the genotype of various specimens according to the returned sequencing results, it was identified that there were 106 cases of deletion of homozygosis, 69 cases of deletion of heterozygosity, 14 cases of wild genotype and 4 cases of special genotype, in which 3 cases of homozygosis (9/9), homozygosis (13/13) and heterozygote (9/13) were respectively selected for in-situ hybridization investigation of miR-205 expression, the results demonstrated that all were negative (Fig. 4).

Analysis of rs3842642 polymorphism in breast cancer and benign breast lesions. DNA was extracted from the paraffin-embedded tissue specimens of breast benign lesions and sent for sequencing. The results of sequencing revealed 119 cases of deletion of homozygosis, 63 cases of heterozygous genotype, 13 cases of wild genotype and 2 cases of special genotype. An association analysis was conducted between obtained results for benign lesions and the results from the breast cancer tissue (Table II). Differences in SNP genotyping between breast cancer and benign lesion tissues were not significant as determined by the $\chi^{2}$ analysis; however, compared with breast cancer tissue, benign lesion tissues exhibited an 
A

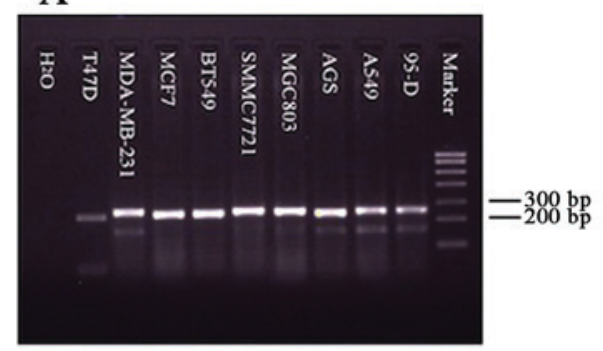

B

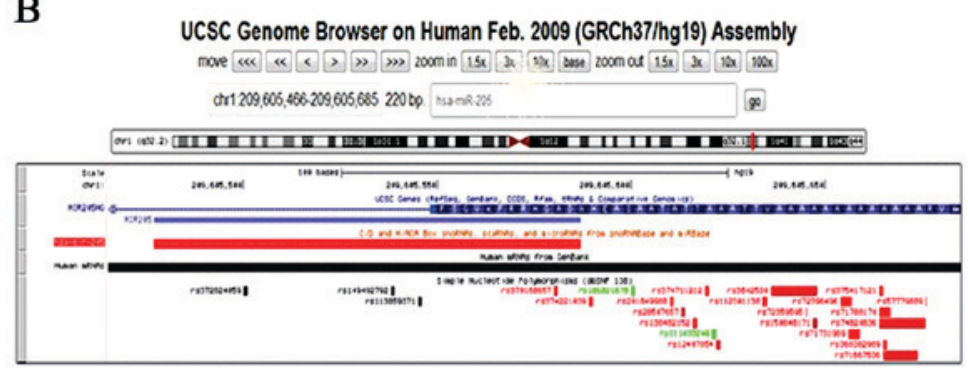

C

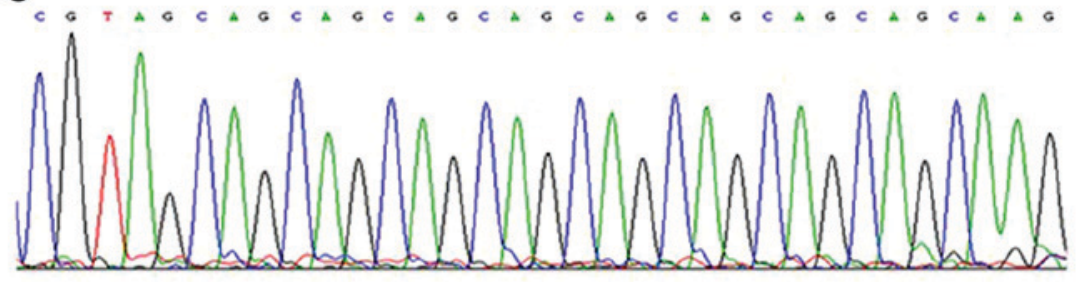

D

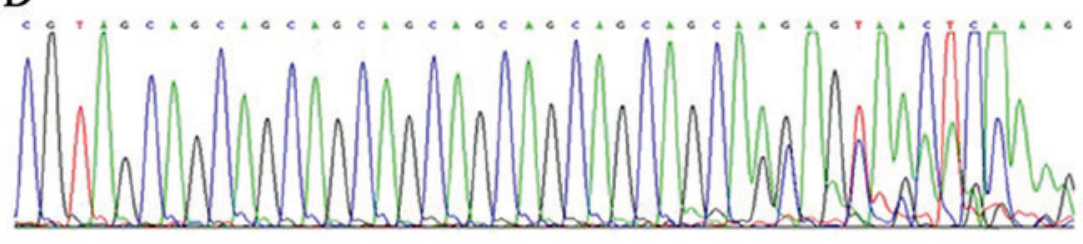

E

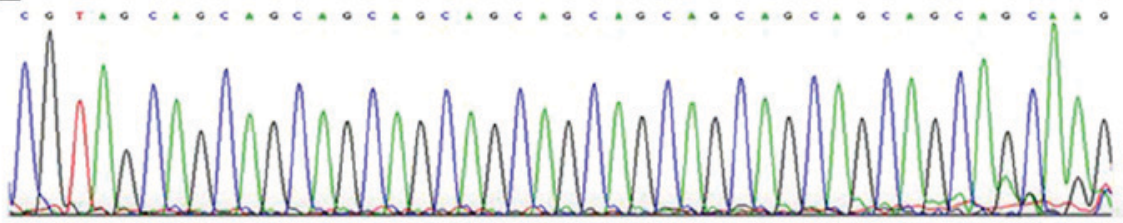

Figure 3. Analysis of polymerase chain reaction amplification and sequencing of miR-205. (A) Amplification of miR-205 gene in 10 cell lines. (B) Gene SNP sites of miR-205; miR-205-rs3842642 commonly existed in these 10 cell lines and was broadly divided into four types: $9 / 9$ homozygous genotype, 13/13 homozygous genotype, 9/13 heterozygous genotype and 7/9 special genotype. Among them, AGS, BT-549 and MCF7 belong to the 9/9 homozygous genotype, 95-D, A549, MGC-803, HEPG2 and SMMC-7721 belong to the 9/13 heterozygous genotype, MDA-MB-231 is the 13/13 homozygous genotype and T47D is the special genotype. (C) rs3842530 9/9 homozygous genotype, CGT(AGC) ${ }_{9}$ AAG/CGT(AGC) ${ }_{9}$ AAG. (D) rs3842530 9/13 heterozygous genotype, CGT(AGC) $)_{9}$ AAG/CGT(AGC) ${ }_{13}$ AAG. (E) rs3842530 13/13 homozygous genotype, CGT(AGC) ${ }_{13}$ AAG/CGT(AGC) ${ }_{13}$ AAG. SNP, single nucleotide polymorphism; miR, microRNA.

increased ratio of 9/9 homozygous genotype. The case number of SNP genotyping corresponding to statistical pathological indicators [estrogen receptor (ER), progesterone receptor (PR), erb-B2 receptor tyrosine kinase 2 (ERBB2)] and association analysis between SNP genotyping and specific pathological indicators were conducted (Table II).

\section{Discussion}

Breast cancer is one of the most common tumors diagnosed in women and an important cause of mortality in breast cancer is the metastasis of cancer cells (15). The epithelial-mesenchymal transition (EMT), as a primary stage of transfer, has been a key area of research into tumor metastasis, and is a process associated with a decrease in E-cadherin expression (16). The expression of E-cadherin is regulated by multiple genes, including nuclear factor- $\kappa \mathrm{B}$ and the matrix metalloproteinase family (17). These genes and their associated cell signaling pathways are influenced by miRNAs, which serve important functions in promoting breast cancer progression by

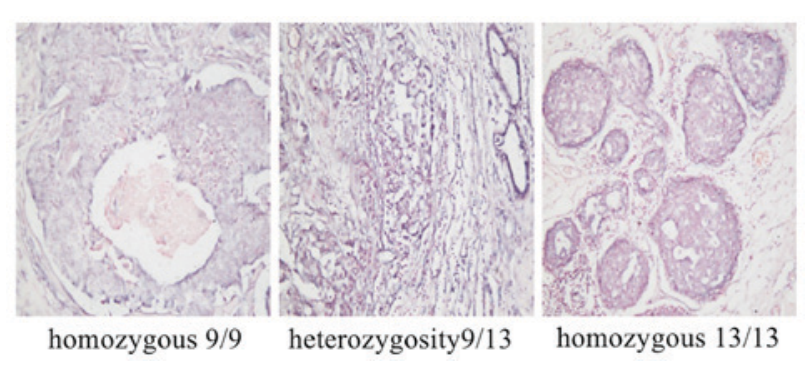

Figure 4. Three SNP Tissue miR-205 in situ hybridization results.

stimulating the EMT process $(18,19)$. MiR-205 is a conserved RNA molecule widely distributed in squamous epithelial tissues of higher living organisms, as its regulation and control of numerous target genes is associated with the onset and development of tumors. Studies investigating miR-205 are primarily focused in the field of tumor biology and have revealed that miR-205 expression may affect the incidence and development of a number of tumors; however, miR-205 serves 
Table II. Single nucleotide polymorphism genotyping and statistical pathological indicators.

rs3842530 polymorphism

\begin{tabular}{|c|c|c|c|c|}
\hline \multirow[b]{2}{*}{ Characteristic } & \multirow[b]{2}{*}{$\mathrm{n}$} & & & \\
\hline & & Homozygous 9/9, n (\%) & Heterozygosity $9 / 13, \mathrm{n}(\%)$ & P-value \\
\hline Breast lesions & & & & 0.362 \\
\hline Benign lesion & 195 & $119(61.03)$ & $76(38.97)$ & \\
\hline Breast cancer & 189 & $106(56.08)$ & $83(43.92)$ & \\
\hline Breast ER expression & & & & $0.014^{\mathrm{a}}$ \\
\hline High & 45 & $19(42.22)$ & $26(57.78)$ & \\
\hline Low & 62 & $41(66.13)$ & $21(33.87)$ & \\
\hline Breast PR expression & & & & 0.112 \\
\hline High & 26 & $11(42.31)$ & $15(57.69)$ & \\
\hline Low & 66 & $40(60.61)$ & $26(39.39)$ & \\
\hline Breast ERBB2 expression & & & & 0.391 \\
\hline High & 18 & $9(50.00)$ & $9(50.00)$ & \\
\hline Low & 77 & $47(61.04)$ & $30(38.96)$ & \\
\hline
\end{tabular}

${ }^{\mathrm{a}} \mathrm{P}<0.05$. ER, estrogen receptor; $\mathrm{PR}$, progesterone receptor; ERBB2, erb-B2 receptor tyrosine kinase.

distinct functions in different types of tumors (20). The expression of miR-205 in breast cancer is generally downregulated; this may be due to the regulation of upstream genes, such as ERBB2 (21), by miR-205 or may be due to SNP variations in the miR-205 sequence. The different functions of miR-205 in breast cancer may be associated with the cellular environment, cancer subtype, origin of the tumor, and the development phase of the tumor (22). The present study revealed that, compared with less metastatic breast cancer cell lines, the expression of miR-205 was decreased in highly metastatic breast cancer cells, suggesting that miR-205 may suppress the metastasis of breast cancer. The results of the present study and the aforementioned studies are in agreement with the results of investigations into the breast cancer cell line and clinical specimens as identified by Wang et al (23) and Liu et al (24).

Numerous different genes regulate the onset and development of breast cancer. MiRNA and various other genes constitute this vast regulatory network and SNPs are present in the various genes involved. SNP variation in these genes may lead to a variation in gene expression, thus affecting breast cancer risk (25). Previous studies have demonstrated that, Breast Cancer Association Consortium rs1045485 and rs1982073 SNPs are associated with an increased risk of breast cancer (26). Wang et al conducted an investigation into 491 Chinese patients with breast cancer and 502 healthy individuals (27), and analyzed the distributional difference of genotypes between them. It was identified that rs10889677 and rs1884444 in the interleukin 23 receptor 3'-UTR region were associated with an increased risk of breast cancer among Chinese women, which also affected the early development of tumors. It was also identified that the cytoskeleton gene IQ motif containing GTPase activating protein 1 (IQGAP) was regulated by miR-124. The results of a study in 1,541 patients with breast cancer and 1,598 healthy controls indicated that the variation in rs1042538 sites in the IQGAP 3'-UTR region affected the binding and regulation of miR-124 to this gene; thus, this may be developed as a potential molecular marker to evaluate the risk and prognosis of patients with breast cancer (28). The results of the aforementioned studies indicate that SNP variation in genes associated with breast cancer is associated with occurrence and development of breast cancer. The important regulatory effect of miRNA in breast cancer has enabled research on miRNA SNP to attract increased attention, with more studies being performed investigating the expression of miR-205 in breast cancer; however, to the best of our knowledge, there have been no studies investigating the association between SNP variation of miR-205 and breast cancer. Following verification that miR-205 is differentially expressed in breast cancer, the present study explored whether SNP variation is associated with the differential expression of miR-205.

In order to improve detection of SNP distribution in miR-205, two lines of lung cancer, gastric cancer and liver cancer cells were investigated along with the breast cancer cell lines. SNP distribution in each cell was detected using DNA sequencing and through this analysis, the presence of rs3842642 was identified and four genotypes within the 10 cell lines that were investigated were also identified: A 9/9 homozygous genotype, a 9/13 heterozygous genotype, a 13/13 homozygously inserted genotype and a special genotype, the high frequency of such sites in cancer cells suggest that they may be associated with tumorigenesis. By detecting the expression of miR-205 in the aforementioned cell lines using RT-qPCR and comparing SNP genotypes in each cell line, it was identified that the expression of miR-205 increased in breast cancer cell lines with an increase of the number of AGC deletions at this site. This association was observed in 4 breast cancer cell lines and it was unclear whether variation at this site significantly affected the expression of miR-205; an increased number of samples are required verify this phenomenon. Therefore, 193 cases of breast cancer specimens were used for sequencing analysis in order to determine the genotype 
of each specimen. The analysis revealed that rs384242 widely existed in these specimens including these four genotypes of the site. The expression of miR-205 in in-situ hybridization was analyzed by separately selecting 3 cases of homozygous deletion, heterozygous deletion and homozygous insertion in breast cancer specimens However the specimen sample size selected was relatively small, thus no significant association was identified in these specimens. A larger sample size may assist in verifying the aforementioned association.

Under normal conditions, miR-205 serves a tumor suppressive function in breast cancer and the expression of miR-205 in breast cancer is inhibited (29). If there is a distributional difference of these three genotypes of rs3842642 in breast cancer specimens and breast benign lesions, the variation in this site may be associated with miR-205 expression and t breast cancer progression. Statistical analysis indicated a P-value $>0.05$ when comparing genotypes between breast cancer and benign specimens, suggesting that there was no significant difference. However, the proportion of 9/9 homozygous deletion in benign breast lesion tissue was slightly increased compared with breast cancer tissue specimens. The differential distribution of distinct genotypes of rs3842642 in breast cancer and benign breast lesions may be identified in a larger sample size.

ER, PR and ERBB2 are the primary indicators of breast cancer used in pathological examinations (30). Notably, the results of the present study demonstrated that there was a significant association between rs3842642 polymorphism and ER expression in breast cancer tissues $(\mathrm{P}=0.014)$; however, there was no association between PR or ERBB2 and breast cancer. The proportion of 9/9 homozygous deletion in the high expression group was increased compared with that of the low expression group. The rs3842642 site of miR-205 should be investigated further in a larger sample of patients; with more in-depth study, this site may become a new molecular marker for the diagnosis of breast cancer.

The expression of miR-205 was detected at the cellular level using RT-qPCR, however, this method was not suitable to detect the expression of miR-205 in paraffin-embedded tissues. In-situ hybridization was adopted to quantify the expression of miR-205 in breast cancer tissues. In the present study, more and less metastatic breast cancer cells were selected for cells grown on coverslips. Cells were collected and fixed and the expression of miR-205 was detected using in-situ hybridization. Subsequently, the expression of miR-205 in various cancer cells was determined by RT-qPCR. The results of RT-qPCR were consistent with those of in-situ hybridization, further confirming the feasibility and creditability of in-situ hybridization to determine the expression of miR-205.

In conclusion, the present study identified the differential expression of miR-205 in breast cancer cells at the cellular level. Decreased expression of miR-205 was associated with metastatic breast cancer and rs3842642 was identified in various tumor cells. The increase in the missing number of AGC repeats at these sites in breast cancer cells was accompanied by an increased expression of miR-205, however this has not been verified in breast cancer specimens. A significant association was identified between genotypes of rs3842642 and ER+ groups in breast cancer tissue specimens, although no significant association was identified between PR and ERBB2 positive groups and breast cancer/breast benign lesions groups.
The present study identified an association between SNP variation of miR-205 and breast cancer, however the association between miR-205 rs3842642 and SNP variation at other sites in breast cancer should be investigated further.

\section{Acknowledgements}

The present study was supported by the Natural Science Foundation of Zhejiang (grant no. LY15H160049) and Zhejiang Province Public Technology Application Research Program (grant no. 2016F81SA700015).

\section{References}

1. Venter JC, Adams MD, Myers EW, Li PW, Mural RJ, Sutton GG, Smith HO, Yandell M, Evans CA, Holt RA, et al: The sequence of the human genome. Science 291: 1304-1351, 2001.

2. Jupe ER, Badgett AA, Neas BR, Craft MA, Mitchell DS Resta R, Mulvihill JJ, Aston CE and Thompson LF: Single nucleotide polymorphism in prohibitin 3' untranslated region and breast-cancer susceptibility. Lancet 357: 1588-1589, 2001.

3. Gudmundsdottir ET, Barkardottir RB, Arason A, Gunnarsson H, Amundadottir LT, Agnarsson BA, Johannsson OT and Reynisdottir I: The risk allele of SNP rs3803662 and the mRNA level of its closest genes TOX3 and LOC643714 predict adverse outcome for breast cancer patients. BMC Cancer 12: 621, 2012.

4. Sun K and Lai EC: Adult-specific functions of animal microRNAs. Nat Rev Genet 14: 535-548, 2013.

5. Mishra PJ, Mishra PJ, Banerjee D and Bertino JR: MiRSNPs or MiR-polymorphisms, new players in microRNA mediated regulation of the cell: Introducing microRNA pharmacogenomics. Cell Cycle 7: 853-858, 2008.

6. Wu C, Gong Y, Sun A, Zhang Y, Zhang C, Zhang W, Zhao G, Zou Y and Ge J: The human MTHFR rs4846049 polymorphism increases coronary heart disease risk through modifying miRNA binding. Nutr Metab Cardiovasc Dis 23: 693-698, 2013.

7. Xu Y, Ma H, Yu H, Liu Z, Wang LE, Tan D, Muddasani R, Lu V, Ajani JA, Wang Y and Wei Q: The miR-184 binding-site rs 8126 $\mathrm{T}>\mathrm{C}$ polymorphism in TNFAIP2 is associated with risk of gastric cancer. PLoS One 8: e64973, 2013.

8. Guo Z, Wang H, Li Y, Li B, Li C and Ding C: A microRNA-related single nucleotide polymorphism of the XPO5 gene is associated with survival of small cell lung cancer patients. Biomed Rep 1: 545-548, 2013.

9. Zhang N, Huo Q, Wang X, Chen X, Long L, Jiang L, Ma T and Yang Q: A genetic variant in pre-miR-27a is associated with a reduced breast cancer risk in younger Chinese population. Gene 529: 125-130, 2013.

10. Gilam A, Edry L, Mamluk-Morag E, Bar-Ilan D, Avivi C, Golan D, Laitman Y, Barshack I, Friedman E and Shomron N: Involvement of IGF-1R regulation by miR-515-5p modifies breast cancer risk among BRCA1 carriers. Breast Cancer Res Treat 138: 753-760, 2013.

11. Linhares JJ, Azevedo M Jr, Siufi AA, de Carvalho CV, Wolgien Mdel C, Noronha EC, Bonetti TC and da Silva ID: Evaluation of single nucleotide polymorphisms in microRNAs (hsa-miR-196a2 rs11614913 C/T) from Brazilian women with breast cancer. BMC Med Genet 13: 119, 2012

12. Smith RA, Jedlinski DJ, Gabrovska PN, Weinstein SR, Haupt L and Griffiths LR: A genetic variant located in miR-423 is associated with reduced breast cancer risk. Cancer Genomics Proteomics 9: 115-118, 2012.

13. Livak KJ and Schmittgen TD: Analysis of relative gene expression data using real-time quantitative PCR and the 2(-Delta Delta C(T)) method. Methods 25: 402-408, 2001.

14. Karolchik D, Barber GP, Casper J, Clawson H, Cline MS, Diekhans M, Dreszer TR, Fujita PA, Guruvadoo L, Haeussler M, et al: The UCSC genome browser database: 2014 update. Nucleic Acids Res 42 (Database Issue): D764-D670, 2014.

15. Mundy GR: Metastasis to bone: Causes, consequences and therapeutic opportunities. Nat Rev Cancer 2: 584-593, 2002.

16. Hay ED: The mesenchymal cell, its role in the embryo, and the remarkable signaling mechanisms that create it. Dev Dyn 233: 706-720, 2005. 
17. Brinckerhoff CE and Matrisian LM: Matrix metalloproteinases: A tail of a frog that became a prince. Nat Rev Mol Cell Biol 3: 207-214, 2002.

18. Zhao X, Lu Y, Nie Y and Fan D: MicroRNAs as critical regulators involved in regulating epithelial-mesenchymal transition. Curr Cancer Drug Targets 13: 935-944, 2013.

19. Ding XM: MicroRNAs: Regulators of cancer metastasis and epithelial-mesenchymal transition (EMT). Chin J Cancer 33: 140-147, 2014

20. Chao CH, Chang CC, Wu MJ, Ko HW, Wang D, Hung MC, Yang JY and Chang CJ: MicroRNA-205 signaling regulates mammary stem cell fate and tumorigenesis. J Clin Invest 124 3093-3106, 2014

21. Adachi R, Horiuchi S, Sakurazawa Y, Hasegawa T, Sato K and Sakamaki T: ErbB2 down-regulates microRNA-205 in breast cancer. Biochem Biophys Res Commun 411: 804-808, 2011.

22. Orang AV, Safaralizadeh R and Hosseinpour Feizi MA: Insights into the diverse roles of miR-205 in human cancers. Asian Pac J Cancer Prev 15: 577-583, 2014.

23. Wang Z, Liao H, Deng Z, Yang P, Du N, Zhanng Y and Ren H: miRNA-205 affects infiltration and metastasis of breast cancer. Biochem Biophys Res Commun 441: 139-143, 2013.

24. Liu J, Mao Q, Liu Y, Hao X, Zhang S and Zhang J: Analysis of miR-205 and miR-155 expression in the blood of breast cancer patients. Chin J Cancer Res 25: 46-54, 2013.

25. Khan S, Greco D, Michailidou K, Milne RL, Muranen TA, Heikkinen T, Aaltonen K, Dennis J, Bolla MK, Liu J, et al: MicroRNA Related Polymorphisms and Breast Cancer Risk. Plos One 9: e109973-e109973, 2014.
26. Michailidou K, Hall P, Gonzalez-Neira A, Ghoussaini M, Dennis J, Milne RL, Schmidt MK, Chang-Claude J, Bojesen SE, Bolla MK, et al: Large-scale genotyping identifies 41 new loci associated with breast cancer risk. Nat Genet 45: 353-361, 2013.

27. Wang L, Liu W, Jiang W, Lin J, Jiang Y, Li B and Pang D: A miRNA binding site single-nucleotide polymorphism in the 3'-UTR region of the IL23R gene is associated with breast cancer. PLoS One 7: e49823, 2012.

28. Zheng H, Song F, Zhang L, Yang D, Ji P, Wang Y, Almeida M, Calin GA, Hao X, Wei Q, et al: Genetic variants at the miR-124 binding site on the cytoskeleton-organizing IQGAP1 gene confer differential predisposition to breast cancer. Int J Oncol 38: 1153-1161, 2011.

29. Wu H, Zhu S and Mo YY: Suppression of cell growth and invasion by miR-205 in breast cancer. Cell Res 19: 439-448, 2009.

30. Bartsch R, Ziebermayr R, Zielinski CC and Steger GG: Triple-negative breast cancer. Wien Med Wochenschr 160: 174-181, 2010.

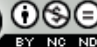

This work is licensed under a Creative Commons Attribution-NonCommercial-NoDerivatives 4.0 International (CC BY-NC-ND 4.0) License. 